\section{AB0389 HIPERTRIGLYCERIDEMIC-WAIST PHENOTYPE: A MARKER OF CARDIOVASCULAR RISK IN RHEUMATOID ARTHRITIS PATIENTS}

I.P. Guzmán Guzmán ${ }^{1}$, O. Zaragoza-García ${ }^{1}$, J.E. Navarro-Zarza², J. MaldonadoAnicacio ${ }^{2}$, N. Castro-Alarcón ${ }^{3}$, I. Parra-Rojas ${ }^{1}{ }^{1}{ }^{1}$ Facultad de Ciencias Químico Biológicas, Universidad Autónoma de Guerrero, Chilpancingo; ${ }^{2}$ Hospital General, Chilpancingo, Guerrero; ${ }^{3}$ Universidad Autónoma de Guerrero, Chilpancingo, Mexico

Background: The risk of cardiovascular disease (CVD) is increased in rheumatoid arthritis (RA); about $50 \%$ of associated deaths in rheumatoid arthritis (RA) are attributed to CVD. ${ }^{1}$ Both traditional cardiovascular risk (CVR) factors and inflammation contribute to this risk. The hyperuricemia (HUC) and metabolic syndrome (MetS) are considered parameters of CVR, both closely related, ${ }^{2}$ and recently the hypertriglyceridemic-waist (HTGW) phenotype has been defined as well a marker to identify HUC in the general population. ${ }^{3}$

Objectives: To determine the prevalence of HTGW phenotype and to evaluate its association with CVR parameters among RA patients.

Methods: HTGW phenotype was defined as waist girth $\geq 102 \mathrm{~cm}$ in male and $>88 \mathrm{~cm}$ in female and TG $>150 \mathrm{mg} / \mathrm{dl}$ in 250 patients diagnosed with RA according to the ACR/EULAR 2010 criteria. The levels of uric acid $>5.5 \mathrm{mg} / \mathrm{dL}$ defined HUC. The MetS and its individual components (NCEP-ATPIII) were evaluated as parameters of $\mathrm{CV}$ risk.

Results: We identified 51 AR patients (20.4\%) whith HTGW phenotype. Adjusting for covariates in a logistic regression model, it was observed that the HTGW phenotype is associated with the presence of HUC $(\mathrm{OR}=6.14, \mathrm{p} \leq 0.001)$, and MetS (OR=5.7, IC95\%, 2.7-12.2, p $\leq 0.001$ ).

Conclusions: HTGW is prevalent in RA patients from southern Mexico. The HTGW phenotype can be considered at low cost marker, used as a tool screening to predict high metabolic risk during the clinical course of the rheumatic disease.

\section{REFERENCES:}

[1] Goshayeshi L, Saber HR, Sahebari M, et al. Association between metabolic syndrome, BMI, and serum vitamin $\mathrm{D}$ concentrations in rheumatoid arthritis. Clin Rheumatol 2012;31:1-7.

[2] Abbasian M, Ebrahimi $\mathrm{H}$, Delvarianzadeh $\mathrm{M}$, et al. Association between serum uric acid (SUA) levels and metabolic syndrome (MetS) components in personnel of Shahroud university of medical sciences. Diabetes Metab Syndr 2016;10:132-6.

[3] Chen S, Guo X, Dong S, et al. Association between the hypertiglyceridemic waist phenotype and hyperuricemia: a cross-sectional study. Clin Rheumatol 2017;36(5):1111-1119.

Acknowledgements: None.

Disclosure of Interest: None declared

DOI: 10.1136/annrheumdis-2018-eular.5906

\section{AB0390 RELATIONSHIP BETWEEN BODY MASS INDEX AND PERSONALITY IN AN EARLY ARTHRITIS COHORT OF PATIENTS}

P. Moreno Fresneda, D. Martínez-Quintanilla Jiménez, N. García Castañeda, T. Velasco Ripoll, A.M. Ortíz García, I. González Álvaro. Rheumatology, HOSP. Universitario La Princesa, Madrid, Spain

Background: According to previously reported data from our early arthritis registry (PEARL, Princess Early Arthritis Register Longitudinal), patients with a higher BMI have higher scores in pain and disability scales. We also described some years ago that, in the same population, the structure of personality explored with the PANAS questionnaire (Positive and Negative Affect Scale) affects some outcomes in arthritis measures. Specifically, higher scores on the negative affect subscale associate higher scores in pain and disability scales.

Objectives: To analyse the relationship between $\mathrm{BMI}$ and the structure of the affect in the PEARL cohort.

Methods: PEARL registry includes patients with early arthritis (less than one year of duration), in whom, sociodemographic, disease related and treatment data are recorded in five protocolised visits. We analysed data from those patients in which the PANAS questionnaire data were available and whose classification, after 2 years of follow-up, were rheumatoid arthritis -according to the 1987 ACR classification criteria - or undifferntiated arthritis. The structure of the affect was evaluated with the PANAS questionnaire administered in one single occasion. This questionnaire is used to evaluate the components of positive (PA) and negative affect (NA). It consists of 20 questions and the score obtained from its administration ranges between 10 and 50 points for each affect. The WHO definition was used for low weight, normal weight, overweight and obesity (BMI <18.5, 18.5-25, $25-30$ or $\geq 30 \mathrm{~kg} / \mathrm{m}^{2}$ respectively). The statistical analysis were performed with
ANOVA and Pearson's correlation tests, as well as multivariate linear regression (including as independent variables sex, age and study level) using the Stata 12.1 software.

Results: We analysed the data of the 71 patients for whom PA values were available and of the 65 patients with NA values available. There was not a stat istically significant relationship observed between PA and NA and BMI when analysed as a continuous variable $(R=-0.12, p=0.25$ and $R=-0.1, p=0.36$, respectively) or as a categorical variable. However, there was a non-significant trend to lower scores for both PA and NA in patients with a higher BMI (figure 1). The multivariate analyses, adjusted for sex, age and study level, also showed no statistically significant relationship between the BMI (analysed as continuous or categorical variable) and the PA and NA; but there was also a non-significant trend that sets a relationship between lower scores of both PA and NA and higher BMI values.
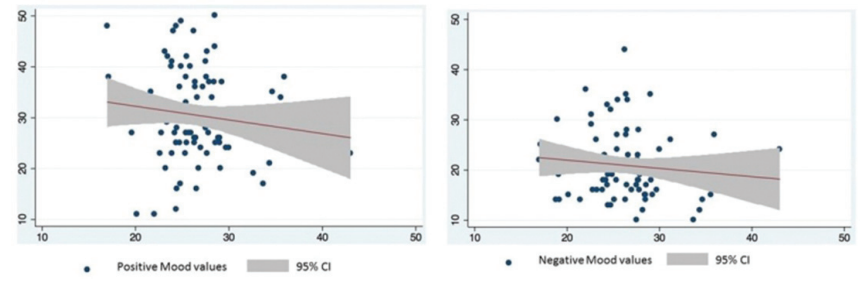

Abstract AB0390 - Figure 1

Conclusions: In our early arthritis registry there is no relationship between the $\mathrm{BMI}$ and the structure of the affect evaluated through the PANAS questionnaire. However, it is necessary to evaluate this relationship in a greater number of patients and in different populations before discarding this relationship definitely.

Disclosure of Interest: None declared

DOI: 10.1136/annrheumdis-2018-eular.3129

\section{$\mathrm{AB} 0391$ \\ CLINICAL, SEROLOGICAL AND TREATMENT ANALYSIS OF RHUPUS SYNDROME: A RETROSPECTIVE MONOCENTRIC STUDY}

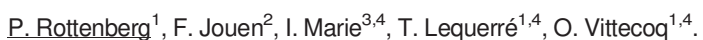

${ }^{1}$ Rheumatology, ${ }^{2}$ Clinical Immunology Laboratory; ${ }^{3}$ Internal Medicine, Rouen University Hospital; ${ }^{4}$ U1234, INSERM, Rouen, France

Background: Rhupus syndrome is a rare condition in which rheumatoid arthritis (RA) features overlap with systemic lupus erythematosus (SLE) manifestations. Disease characterisation tends to improve, but some uncertainties remain, and therapeutic management has still to be defined.

Objectives: We aimed to perform a study of the patients followed at the Rouen University Hospital with rhupus diagnosis using both recent criteria and expert diagnosis, in order to perform an analysis of the clinical, radiological and biological characteristics, and to report the therapeutic procedures carried out.

Methods: We performed a retrospective research work covering a period of 10 years, using diagnosis code extraction for both RA and SLE from database of the medical information department, as well as biological data extraction concerning ACPA testing, anti-dsDNA and anti-RNP antibodies. Patients satisfying both ACR/EULAR 2010 and SLICC 2012 classification criteria for RA and SLE respectively, or with an expert diagnosis of rhupus were included. A screening of medical records was performed to collect clinical, biological and treatment data for each patient.

Results: Sixteen patients were identified and 12 fulfilled both classification criteria. RA most often preceded rhupus, and clinical analysis found a predominant articular pattern at initial and established disease, with erosive arthropathy $(n=11)$. Skin involvement was the most frequent associated manifestation $(n=12)$. Among other associated manifestations, serious events were reported, including active glomerulonephritis $(n=2)$, ischaemic stroke $(n=1)$ and myocardial infarction $(n=1)$. Immunological profiles showed positivity for $(n=14)$, anti-dsDNA $(n=9)$ and ACPA $(n=9)$. Ten patients required biological DMARD, in addition to conventional treatment. All types of RA approved bDMARD were used. Rituximab was the most prescribed $(n=9)$ and the most effective, with sustained response in 5 patients.

Conclusions: Rhupus is a rare condition, with predominant articular pattern, but serious SLE-related manifestations can occur. In rhupus refractory to conventional treatment, biologics, and particularly rituximab, are a promising therapeutic approach.

Disclosure of Interest: None declared

DOI: 10.1136/annrheumdis-2018-eular.2432 